\title{
De-noising Method based on Median Filter and Wavelet Transform
}

\author{
Weibo $\mathbf{Y u}^{12}$ \\ College of Electrical and Electronic Engineering, Changchun University of Technology,Changchun,Jilin, \\ 130012, China \\ E-mail: yu_weibo@126.com
}

\section{Fangxue Liu}

College of Electrical and Electronic Engineering, Changchun University of Technology, Jilin, 130012, China

E-mail: $131258158780163 . \mathrm{com}$

\section{Liming Zheng}

College of Electrical and Electronic Engineering, Changchun University of Technology,Jilin, 130012, China

E-mail: zhengliming9410163.com

\section{Dongmei Wang}

College of Electrical and Electronic Engineering, Changchun University of Technology, Jilin, 130012, China

E-mail: wangdongmei@mail.ccut.edu.cn

As to the hybrid noise characteristic, a mixed de-noising method is hereby proposed in combination with the median filter and the wavelet transform. Firstly, conduct the median filter to the pending image; then apply an improved threshold function to the wavelet transform (WT) to eliminate noise; finally, adopt Signal Noise Ratio (SNR) to evaluate the image de-noising effect. The experiment results show that de-noising effect of the new algorithm is more excellent than the traditional wavelet transform and can eliminate hybrid noise so that the image quality can be improved effectively.

CENet2015

12-13 September 2015

Shanghai, China

\section{${ }^{1}$ Speaker \\ ${ }^{2}$ Corresponding Author}




\section{Introduction}

The image contains various kinds of noise due to the influence of complicated background, illumination and acquisition system. The existence of image noise leads to the image quantity reduction, which has exerted strong impact on the accuracy of industrial inspection, face identification and feature detection [1].

In this sense, it is a fatal issue of image preprocessing to eliminate noise. The image noise mainly behaves as Gaussian and Impulse noise [2]. Median filter has good inhibiting effect on the impulse noise, well protects the detail information such as margin and acute angle, etc [3]; but the inhibition ability to Gaussian distribution is weaker. The de-noising method based on WT can focus on detail area of the image, magnify small change, well inhibit the noise of Gaussian distribution, but partial impulse noise will be left [4], as a result, combine median filter and wavelet transform together to realize image de-noising.

\section{Theoretical Basis}

\subsection{Median filtering}

The median filter is a typical nonlinear spatial filter method for removing noise. It is the main idea that a point in value of the digital image is replaced by the median value of each point in the neighborhood [5]. The mathematical definition of median filter is shown as follows: given an array $A=\left[x_{1}, x_{2}, \ldots, x_{n}\right], x_{i}$ is the gray value of the pixel, as shown in Formula (2.1).

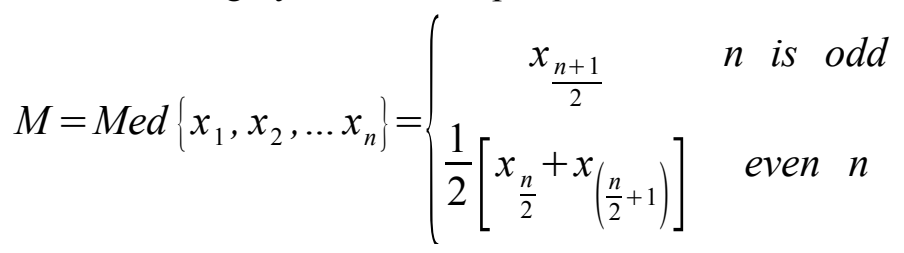

\subsection{Filter Window Size Selection}

The size of the median filter window affects the filter performance. $p_{k}$ is the intensity of noise interference. If $p_{k}$ is small, the image details can be retained well. If $p_{k}$ is large, it performs well in the filter ability and makes the image details blurred. With this principle, if a square window is selected for filtering, the window length is shown as follows.

$$
l_{k}=\left\{\begin{array}{lc}
0, & p_{k}<0.2 \% \\
3, & 0.2 \% \leqslant p_{k} \leqslant 3 \% \\
5, & P_{k}>3 \%
\end{array}\right.
$$

\subsection{Wavelet Transform}

Basically, the wavelet transform through space and frequency of the local transformation, extracting information from signals [6]. A detailed analysis of the signal can be used for image compression and filtering, etc. Wavelet transform de-noising is used to complete the image wavelet decomposition, threshold and reconstruct wavelet coefficient. The digital image basically can be viewed as a finite energy signal $f(x, y)$. If $c_{j, m, n}(x, y)$ is the scale function, $\varphi_{j, m, n}^{l}(x, y)$ is the translation base function, and $l \in\{1,2,3\}$, the wavelet transform of discrete function $f(x, y)$ of $\mathrm{M} * \mathrm{~N}$ is shown as follows [7]: 


$$
\begin{aligned}
& W_{c}\left(j_{0}, m, n\right)=\frac{1}{\sqrt{M N}} \sum_{x=0}^{M-1} \sum_{y=0}^{N-1} f(x, y) c_{j_{0}, m, n}(x, y) \\
& W_{\varphi}^{l}(j, m, n)=\frac{1}{\sqrt{M N}} \sum_{x=0}^{M-1} \sum_{y=0}^{N-1} f(x, y) \varphi_{j, m, n}^{l}(x, y)
\end{aligned}
$$

Where $j_{0}$ is an arbitrary scale. It is usually 0 . And $M=N=2^{j}, j=0,1, \ldots J-1$, $m=n=0,1, \ldots 2^{j}-1$. (2.3) and (2.4) are wavelet transform of two-dimensional signal. $W_{c}$ stands for the approximation in the scale of $j . W_{\varphi}^{1}, W_{\varphi}^{2}, W_{\varphi}^{3}$ denotes details of vertical, horizontal, diagonal. $W_{c}\left(j_{0}, m, n\right)$ and $W_{\varphi}^{l}$ refer to the wavelet coefficient.

\section{Median Filter and Wavelet Transform Hybrid De-noising Algorithm}

\subsection{Basic Idea of the Algorithm}

Firstly, remove the salt $\&$ pepper noise with median filter to obtain images only containing the Gaussian noise; then eliminate Gaussian noise by using wavelet transform. The wavelet transform de-noising is adopted to remove the non-useful information. The non-useful often exists in high-frequency part. The main information is mostly distributed in low-frequency subband upon the wavelet decomposition. To avoid destructing main information, the highfrequency wavelet coefficients are disposed by threshold and $5 * 5$ median filter. Finally, the wavelet coefficients of each sub-band are reconstructed by wavelet, so we obtain the de-noising image.

\subsection{Determination of Decomposition Level}

The wavelet decomposition of images is that images are decomposed by selecting appropriate wavelet basis and layers of the wavelet decomposition [8]. It is obviously different to the analysis of different signals with different wavelet basis functions, so it is critical to choose the wavelet basis function. According to principles of wavelet basis selection and the experience of image preprocessing, Db3 wavelet basis is selected.

As to the two-dimensional discrete wavelet transform, we get four sub-images: $W_{c}, W_{\varphi}^{1}, W_{\varphi}^{2}$, $W_{\varphi}^{3}$ in the wavelet decomposition of each layer. $W_{c}$ is only decomposed in the decomposition of the next layer. We obtain another four sub-images until it predetermines the number of decomposition [9]. Four sub-images are produced in the wavelet decomposition of every layer. The cascaded filters increase with the decomposition layers, which leads to the signal shifts to get enlarged with less and less information of each high frequency sub-graph [10]; therefore, the three-layer wavelet decomposition is selected.

\subsection{Improvement of Threshold Function}

After the wavelet decomposition, most energy concentrates on the low-frequency wavelet coefficient and few energy distracts in high-frequency wavelet coefficient. The wavelet coefficient value of useful signal is larger than that of noise. To choose the thresholds directly affecting the image quality, the selection of threshold function is the different processing methods of wavelet coefficients that are larger or smaller threshold.

Hard threshold function does well in reserving the local feature, but its discontinuity can make the reconstructed image to produce the gibbes, etc. The treatment of soft threshold is relatively smooth; but the error in estimating the wavelet coefficients makes the image edge to be fuzzy. To avoid the gibbes and overcome the disadvantage of soft threshold in de-noising, we form a new threshold function. The expressions are given as follows.

$$
\hat{W}_{j, m, n}=\left\{\begin{array}{c}
\operatorname{sign}\left(W_{j, m, n}\right) \mu\left(\left|W_{j, m, n}\right|-\alpha \lambda\right)(0 \leq \alpha \leq 1) \\
0
\end{array}\right.
$$




$$
\mu=\left\{\begin{array}{cc}
1 & \left|W_{j, m, n}\right| \geq \lambda \\
\frac{\lambda}{\lambda-\lambda_{0}}, & \lambda_{0} \leq\left|W_{j, m, n}\right|<\lambda \\
0 & \left|W_{j, m, n}\right|<\lambda_{0}
\end{array}\right.
$$

Where sign (.) is sign function, $\lambda$ is threshold. Due to limitations of soft and hard functions, the weight function $\mu$ is used to adjust the soft and hard functions. It not only preserves the edge details but also does not lose useful information. We select $\lambda_{0}=0.6 \lambda$ through experiments.

\subsection{New Threshold Determination}

According to (3.1) and (3.2), it is important to select the threshold. If the threshold is too large, the most wavelet coefficients are set to zero, which leads to loss of too many detail signals. If the threshold is too small, it can not reach the expected effect. The fixed threshold method is used to estimating threshold.

$$
\lambda=\sigma \sqrt{2 \ln N}
$$

Where $N$ is the number of wavelet decomposition coefficients all scales, $\sigma$ is the noise variance. The valid signal wavelet transform is still clear on large scale. The threshold varies with different scales. The threshold varies inversely with the scale. Eq. (3.4) is an improved threshold function. According to (3.4), the threshold is a fixed threshold if $j=1$, while the threshold is changing with the scale of $j$ if $j>1$.

$$
\lambda_{j}=\sigma \sqrt{2 \log N} / \ln (e+j-1)
$$

\section{Experimental Results and Analysis}

\subsection{Color Image De-noising}

We conduct an experiment on standard image in California Institute of Technology Library. The image added noise of different intensities is processed with median filter, wavelet transform and hybrid de-noising algorithm. The SNR shown in Table1 is used to evaluate results. Fig. 1 shows the results of different de-noising algorithms. Fig. (1-c) and Fig. (1-d) are not clearer than Fig. (1-e) and Fig. (1-f), even lose some important details. There is a small part of vagueness or distortion in Fig. (1-e). Fig. (1-f) performs better than Fig. (1-e) in reserving the original image information.

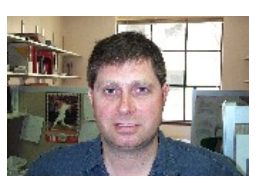

(a)

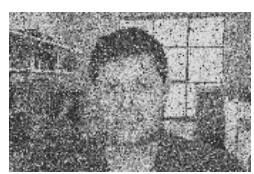

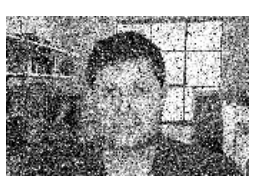

(b)

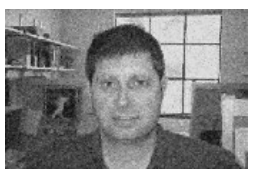

$(\mathrm{e})$
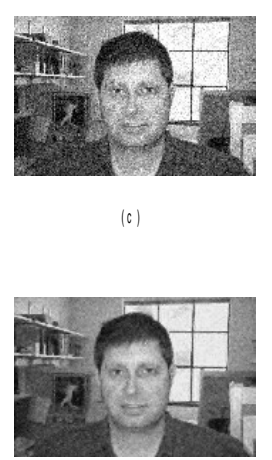

Figure 1 : Results of Different De-noising Algorithms: (a) Original Image; (b) Noisy Image; (c) Median Filter; (d) Wavelet Transform De-noising; (e) Wavelet Transform and 3*3 Median Filter; (f) Wavelet Transform and 5*5 Median Filter $(\sigma=0.05, \rho=0.05)$ 


\begin{tabular}{|c|c|c|c|c|c|c|c|c|c|}
\hline \multirow{3}{*}{$\begin{array}{l}\text { Noise intensity } \sigma / \\
\text { density } \rho\end{array}$} & \multirow{3}{*}{ No de-noising } & \multicolumn{2}{|c|}{ Median filter } & \multicolumn{2}{|c|}{$\begin{array}{l}\text { Wavelet } \\
\text { transform }\end{array}$} & \multicolumn{4}{|c|}{$\begin{array}{l}\text { Combining wavelet transform and } \\
\text { median filtering }\end{array}$} \\
\hline & & \multirow[b]{2}{*}{$3 * 3$} & \multirow[b]{2}{*}{$5 * 5$} & \multirow{2}{*}{$\mathrm{Db} 3$} & \multirow{2}{*}{$\mathrm{Db} 6$} & \multicolumn{2}{|c|}{ Db3 } & \multicolumn{2}{|c|}{$\mathrm{Db} 6$} \\
\hline & & & & & & $3 * 3$ & $5 * 5$ & $3 * 3$ & $5 * 5$ \\
\hline $0.05 / 0.05$ & 7.97 & 15.35 & 17.77 & 8.06 & 8.02 & 17.80 & 18.96 & 17.80 & 18.91 \\
\hline $0.2 / 0.2$ & 3.52 & 8.64 & 11.97 & 3.63 & 3.60 & 12.26 & 14.28 & 12.26 & 13.54 \\
\hline $0.02 / 0.2$ & 6.35 & 16.50 & 18.82 & 6.45 & 6.43 & 19.15 & 19.85 & 19.15 & 19.29 \\
\hline $0.2 / 0.02$ & 4.81 & 10.43 & 13.54 & 4.91 & 4.93 & 13.03 & 15.07 & 13.03 & 15.18 \\
\hline
\end{tabular}

Note: In the combination of de-noising, $3 * 3$ said both before and after use the same filter; $5 * 5$ said in front of the $3 * 3$ filter, $5 * 5$ filter was used in high frequency coefficient.

Table 1 : Performance of Different Filters (Unit dB)

According to Table 1, the effect of the combined algorithms is better than the traditional median filter and the wavelet de-noising. The SNR value of $\mathrm{Db} 3$ wavelet is better than that of Db6 wavelet. The SNR value is higher when the median filter is $5 * 5$ in high frequency coefficient part. Taking the image added with Gaussian noise of 0.05 and salt \& pepper of 0.05 , the SNR value of combined algorithm reaches $18.96 \mathrm{~dB}$ that increases by $3.61 \mathrm{~dB}$ than traditional median filter of $3 * 3$, and $10.9 \mathrm{~dB}$ than the single wavelet de-noising.

\subsection{Standard Gray Image De-noising}

Moreover, we conduct an experiment on Lena standard test image in Matlab image library. The image added noise of different intensities is processed with median filter, wavelet transform and hybrid de-noising algorithm. The SNR shown in Table 2 is used to evaluate the results. Fig. 2 shows the results of different algorithms. As the results in Fig. 2, the single de-noising method cannot preserve the edge information of image.
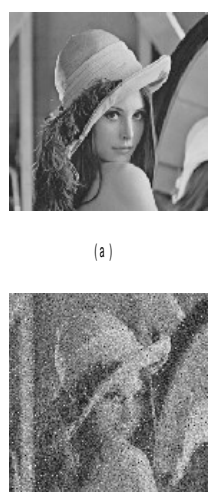

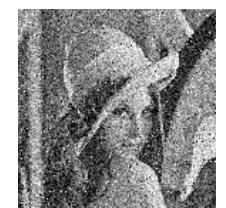

(b)

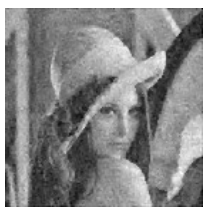

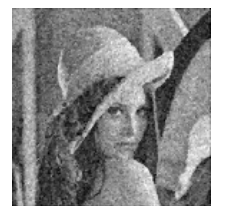

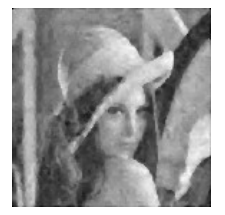

Figure 2 : Results of Different De-noising Algorithms of Lena: (a) Original Image; (b) Noisy Image; (c)Median Filter; (d) Wavelet Transform De-noising; (e) Wavelet Transform and 3*3 Median Filter; (f)Wavelet Transform and 5*5 Median Filter $(\sigma=0.02, \rho=0.02$ )

\begin{tabular}{|c|c|c|c|c|c|c|c|c|c|}
\hline \multirow{3}{*}{$\begin{array}{l}\text { Noise intensity } \sigma / \\
\text { density } \rho\end{array}$} & \multirow{3}{*}{$\begin{array}{l}\text { No de- } \\
\text { noising }\end{array}$} & \multicolumn{2}{|c|}{ Median filter } & \multicolumn{2}{|c|}{$\begin{array}{l}\text { Wavelet } \\
\text { transform }\end{array}$} & \multicolumn{4}{|c|}{$\begin{array}{l}\text { Combining wavelet transform } \\
\text { and median filtering }\end{array}$} \\
\hline & & \multirow{2}{*}{$3 * 3$} & \multirow{2}{*}{$5 * 5$} & \multirow{2}{*}{$\mathrm{Db} 3$} & \multirow{2}{*}{ Db6 } & $\mathrm{Db} 3$ & & Db6 & \\
\hline & & & & & & $3 * 3$ & $5 * 5$ & $3 * 3$ & $5 * 5$ \\
\hline $0.05 / 0.05$ & 6.89 & 13.97 & 16.21 & 6.90 & 6.91 & 16.25 & 17.35 & 16.25 & 16.78 \\
\hline $0.2 / 0.2$ & 2.68 & 7.31 & 10.44 & 2.82 & 2.80 & 11.05 & 13.20 & 11.05 & 12.63 \\
\hline $0.02 / 0.2$ & 5.70 & 15.09 & 17.01 & 5.74 & 5.77 & 17.29 & 17.87 & 17.29 & 18.13 \\
\hline $0.2 / 0.02$ & 3.72 & 9.04 & 11.94 & 3.80 & 3.83 & 12.45 & 14.21 & 12.45 & 14.16 \\
\hline
\end{tabular}

Note: As shown in Table 1

Table 2: SNR of Different Filters for Lena (Unit dB)

As shown in Table 2, the de-noising effect of combined algorithm is better than others. The median filter of $5 * 5$ is better than $3 * 3$ in combination with the wavelet basis of Db3 and high frequency. Taking the identical noise in the former experiment, the SNR value of combined 
algorithm reaches $17.35 \mathrm{~dB}$, increased by $24.2 \%$ than the traditional median filter of $3 * 3$, and much larger than the single wavelet de-noising.

\section{Conclusion}

In order to solve the problem that single de-noising method cannot effectively remove hybrid noise, this paper proposes a method in combination with the median filter and the wavelet transform. Firstly, the image is processed with the median filter. Then an improved threshold function and median filter are used for the high-frequency coefficients obtained by the wavelet decomposition; finally, the de-noising image is obtained by wavelet reconstruction for all wavelet coefficients. Experimental results show that the proposed method can make up for the shortcomings of single de-noising method, and this method can not only eliminate the interference of hybrid noise, but also reserve the edge profile and details of image. In addition, this method can make full use of its advantages; thus it is feasible.

\section{References}

[1] L. -L. Xiang, Z. Liu, J. Qi, Y. Yang. Method of Video Image De-Noising Based on Mixed Filter [J]. Journal of Jilin University (Information Science Edition). 31(3): 266-271(2013) (In Chinese)

[2] V. Roy, S. Shukla. Performance Evaluation of Different Image Denoising Methods [J].International Journal of Electronics and Computer Science Engineering. 1(3): 1757-1764(2012)

[3] X. -H.Wan. Image Denoising Based on Wavelet Transform and Median Filter [J]. Journal of Beihua University (Natural Science). 13(3): 352-355(2012) (In Chinese)

[4] P. -T. Zhang, J.-H. Liu. Application of CWT in Mechanical Fault Feature Extraction [J]. Journal of Jilin University (Information Science Edition). 32(2): 172-176( 2014) (In Chinese)

[5] Y. -Q. Zhao. Multi-level de-noising and enhancement method based on wavelet transform for mine monitoring [J]. International Journal of Mining Science and Technology. 23(1): 163-166(2013) (In Chinese)

[6] A. Jaiswal, J. Upadhyay, A. Somkuwar. Image de-noising and quality measurements by using filtering and wavelet based techniques [J]. AEUE - International Journal of Electronics and Communications. 68(8): 699-705(2014)

[7] S. K. Chowdhury, A.D. Nimbarte. Comparison of Fourier and wavelet analysis for fatigue assessment during repetitive dynamic exertion [J]. Journal of Electromyography and Kinesiology. 25(2): 205-213(2015)

[8] A. S. Virk, M. Kaur, L. Passrija. Performance Evaluation of Image Enhancement Techniques in Spatial and Wavelet Domains [J]. International Journal of Computers \& Technology. 3(1): 162166(2012)

[9] X. -W. Wang, S. -L. Wang, K. Li. Infrared image enhancement based on pseudo median filter and wavelet transform [J]. Laser\& Infrared. 43(1): 90-93(2013) (In Chinese)

[10]Y. -H. Zhang, F. -Q. Wang, X. -D. Song, Q.-H. Chen. A De-noising Method of Fingerprint Image Based on Mid-Value Filtering and Wavelet Transform [J]. Microcomputer Information. 26(21): 226227+130(2010) (In Chinese) 